Portland State University

PDXScholar

\title{
Differential Effects of Buprenorphine/Naloxone Vs. Methadone on Quality of Life Over Time: A Subset of The BRAVO Study (Buprenorphine to Improve HIV Care Engagement and Outcomes: A Randomized Trial)
}

Ali Mirzazadeh Javaheri Portland State University

Follow this and additional works at: https://pdxscholar.library.pdx.edu/honorstheses

Part of the Community Health and Preventive Medicine Commons, and the Other Medical Sciences Commons

Let us know how access to this document benefits you.

\section{Recommended Citation}

Mirzazadeh Javaheri, Ali, "Differential Effects of Buprenorphine/Naloxone Vs. Methadone on Quality of Life Over Time: A Subset of The BRAVO Study (Buprenorphine to Improve HIV Care Engagement and Outcomes: A Randomized Trial)" (2020). University Honors Theses. Paper 953.

https://doi.org/10.15760/honors.976

This Thesis is brought to you for free and open access. It has been accepted for inclusion in University Honors Theses by an authorized administrator of PDXScholar. Please contact us if we can make this document more accessible: pdxscholar@pdx.edu. 
Differential Effects of Buprenorphine/Naloxone Vs. Methadone on Quality of Life Over Time: A Subset of The BRAVO Study (Buprenorphine to Improve HIV Care Engagement and Outcomes: A Randomized Trial)

by

Ali Mirzazadeh Javaheri

An undergraduate honors thesis submitted in partial fulfillment of the requirements for the degree of

Bachelor of Science

in

University Honors

and

Science

Thesis Adviser

Dr. Todd Korthuis, MD, MPH

Portland State University

2020 


\section{- Abstract}

Health-related Quality of Life (QoL) is generally poorer in individuals with opioid use disorder (OUD) in comparison with those suffering from other chronic diseases. ${ }^{11}$ Little is known about the difference each buprenorphine/naloxone (BUP/NX) or methadone maintenance therapy (MMT) may make in the QoL for the HIV-infected people with OUD who are new to HIV care. The objective of this study is to evaluate the difference in the impact that BUP/NX and MMT treatment delivery models make on the QoL of the HIV-infected participants with OUD in Vietnam.

This study was a subset of the BRAVO study, buprenorphine to improve HIV care engagement and outcomes: a randomized trial. This study analyzed the QoL data from 254 participants (121 participants belonging to the BUP/NX group), excluding 30 due to lack of follow-up assessments, based on EUROQOL's EQ-5D instrument standards.

Examination of the 5-level Likert scale of the five dimensions of mobility, self-care, usual activities, pain/discomfort, and anxiety/depression over 12 months suggests both treatment delivery models tend to improve QoL for the patients of such cohort, but not in all dimensions. Self-care and activity level were two of the dimensions which did not seem affected by either of the treatment delivery models. And the BUP/NX randomized group demonstrated more favorable improvements in the mobility and pain dimensions while anxiety levels were improved almost equally in both groups.

Keywords: Quality of life, health related quality of life, buprenorphine, naloxone, methadone, HIV, Vietnam

\section{- Background}

Individuals with opioid use disorder (OUD) are at a higher risk for transmission of viral infections like human immunodeficiency virus (HIV), HBV, and HCV due to both unsafe sexual activity and sharing injection equipment such as needles. ${ }^{1}$ It has also been documented that this population is more prone to overdose. ${ }^{9}$ In Vietnam, the number of people who use drugs (PWUD) increased by $70 \%$ between 2000 and $2004 .{ }^{2}$ By 2006, the HIV prevalence rate among PWUD increased to 70-85\% in several areas. ${ }^{3}$ The opioid crisis and its linkage to HIV and other viral infections continue. Moreover, injection drug use (IDU) adversely impacts engagement across the continuum of HIV care: being diagnosed with HIV promptly, linking to HIV care, receiving, and adhering to antiretroviral therapy (ART), staying in HIV care, and achieving HIV viral suppression.

Methadone maintenance therapy (MMT) can both decrease the risk of transmitting HIV and support access, utilization, and outcomes of HIV-related services in the case of injection driven HIV epidemics. "MMT is a long-term and slow-onset substitution therapy, which needs complete medication adherence to achieve optimal outcomes ... and it has been well documented that patients' adherence to MMT was not optimal and the retention rate was just $40 \%$ [between 2015 and 2018 in Vietnam and few other countries]". ${ }^{5}$ Although MMT is actively implemented in Vietnam, only $65.6 \%$ of individuals under treatment did not have optimal adherence to MMT. ${ }^{5,6}$ 
Another course of action for opioid maintenance therapy is implementing use of buprenorphine/naloxone (BUP/NX). BUP/NX, a partial opioid agonist, is a safer and less risky treatment in comparison to methadone, a full opioid agonist, which requires close monitoring. ${ }^{7}$ $\mathrm{BUP} / \mathrm{NX}$ reduces overdose risk and deaths as MMT does. It, however, has less potential for abuse, and causes less analgesia and euphoria symptoms compared with Methadone. ${ }^{9}$ Due to BUP/NX's "ceiling effect", respiratory suppression will not increase after reaching a certain threshold. ${ }^{10}$ However, despite the positive qualities of BUP/NX, patients on BUP/NX are less likely to be retained in treatment compared to those treated with MMT.

Nonetheless, training HIV providers to manage OUD with buprenorphine/naloxone (BUP/NX) during HIV clinic visits has the potential to expand access to opioid dependence treatment while increasing engagement in HIV care.

Health-related Quality of Life (QoL) is generally poorer in individuals with OUD in comparison with those suffering from other chronic diseases. ${ }^{11}$ Little is known about the difference each BUP/NX or MMT may make in the QoL for the HIV-infected people with OUD who are new to HIV care. The objective of this study is to evaluate the difference in the impact that BUP/NX and MMT treatment delivery models make on the QoL of the HIV-infected participants with OUD. The results of this study are to help researchers and clinicians assess relative QoL related differences in each treatment delivery method.

- Methods

a. Study design and setting

This study reports the effect of BUP/NX treatment model vs. MMT treatment model on quality of life over time for individuals participating in the Buprenorphine to Improve HIV Care Engagement and Outcomes (BRAVO) Randomized Trial (ClinicalTrials.govNCT01936857).

The BRAVO study enrolled HIV infected individuals with moderate to severe OUD were randomized to receive HIV clinic based BUP/NX versus referral for MMT for treatment of OUD at six Vietnamese HIV clinics from 2015 to 2019.

The study sites were four HIV clinics in Hanoi, one in Thanh Hoa, and one in Bac Giang; all of which are associated with MMT and HIV testing and counselling centers. Additional outreach recruitment, non-medication-related study procedures and linkage of participants to participating HIV clinic sites for clinical management were carried out by the research coordinating center at Hanoi Medical University (HMU).

This study was conducted in partnership with HMU, the Providence AIDS Control (PAC) authorities of Hanoi, Thanh Hoa, and Bac Giang, the Vietnam National Institute of Mental Health, and Oregon Health and Science University (OHSU). ${ }^{\mathrm{n}+1}$

b. Participants

HIV positive participants who had a current moderate to severe DSM-5 OUD diagnosis, tested positive for opioids through urine drug screening were included in the study. These participants 
had interest in receiving treatment for opioid dependence and were at or above the age of 18 . If female, these participants were willing to practice effective methods of birth control as the study inclusion criteria suggested.

Participants were excluded if they had a known hypersensitivity to buprenorphine or naloxone, AST \& ALT > 5x the upper limit, serious medical or psychiatric illness in past 30 day (e.g. opportunistic infection, psychosis) that precludes safe participation in the opinion of study physician, and received MMT within 30 days of consent. Exclusion criteria also included those who were pregnant or breastfeeding. In addition, known vulnerable populations (children, pregnant women, decisionally-impaired adults, and prisoners) were excluded.

c. Measures

The QoL data was collected and analyzed based on EUROQOL's EQ-5D instrument standards, a preference-based measure of health status that is used in clinical trials and population studies. In this standard method of measurement, five dimensions of mobility, self-care, usual activities, pain/discomfort, and anxiety/depression are examined to measure health-related quality of life. ${ }^{8}$ Each dimension is a 5-level Likert scale; the values are non-arithmetic. Each value from 1-5, respectively represents, no problems, slight problems, moderate problems, severe problems and unable to.

The number of enrolled participants with QoL data was 284; 30 of whom were excluded in the data analysis due to lack of follow-up assessments. The remaining randomized sample included 121 participants belonging to the BUP/NX group and 133 participants belonging to the MMT referral group.

Participants reported QoL during quarterly survey assessments through 12 months follow-up (i.e. 5 possible responses per participant across time).

d. Data analysis

Data analysis and visualization were done through the Microsoft Excel and Tableau software. Five graphs were made for each QoL dimension for both treatment models. 


\section{- Data}

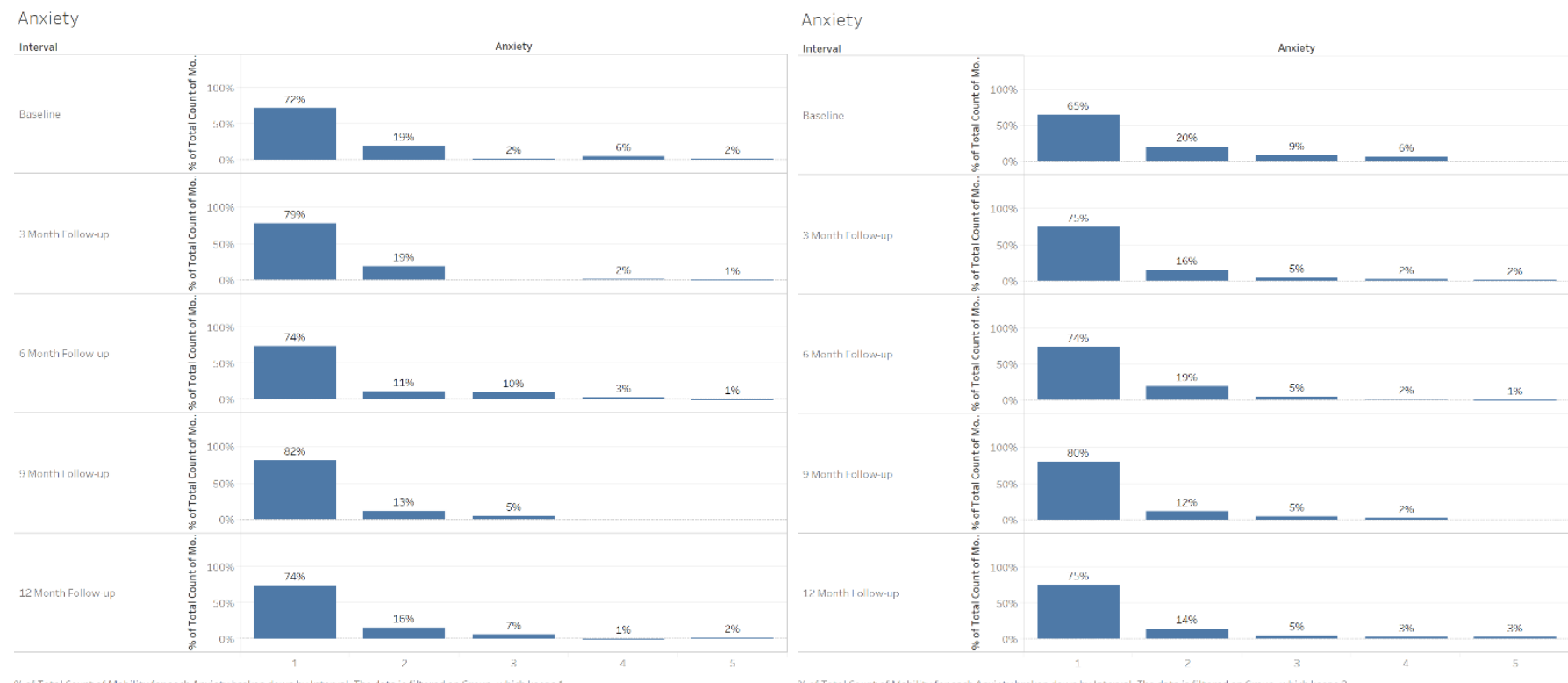

Figure 1. EQ-5D Anxiety dimension by treatment group. Group 1 (BUP/NX) is displayed on the left along with group 2 (MMT) on the right. Slight improvement in Anxiety levels towards the middle of the study followed by a decreasing value towards the end of the study is observed in BUP/NX group. The MMT group shows steady, yet slight, improvement in anxiety. The level of difference in improvement towards the end of study is small.

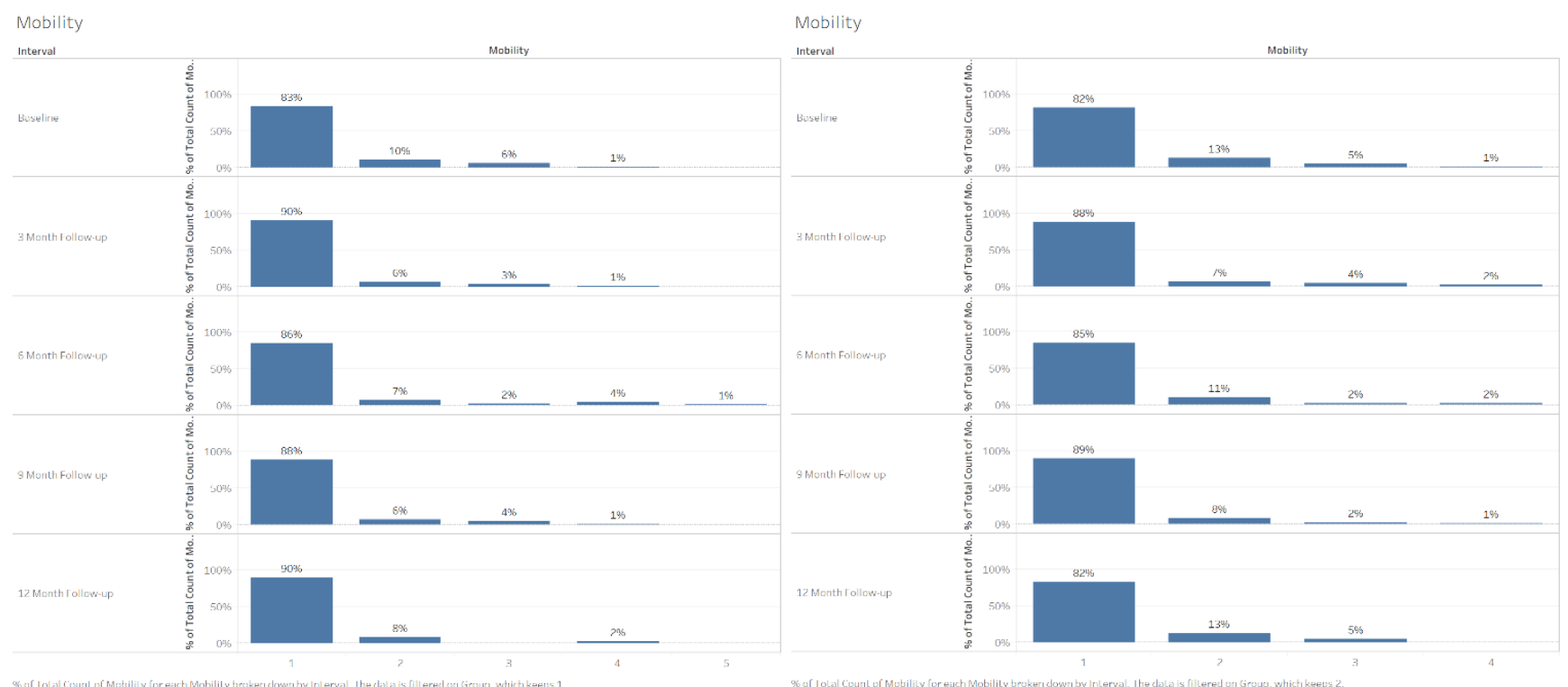


Figure 2. EQ-5D Mobility dimension by treatment group. Group 1 (BUP/NX) is displayed on the left along with group 2 (MMT) on the right. Slight and quiet steady improvement in mobility levels is observed in BUP/NX group, through the course of study. While MMT group shows slight improvement towards the middle of study followed by a diminished level almost equal to the beginning values. The results suggest better mobility in the BUP/NX group by the end of study.

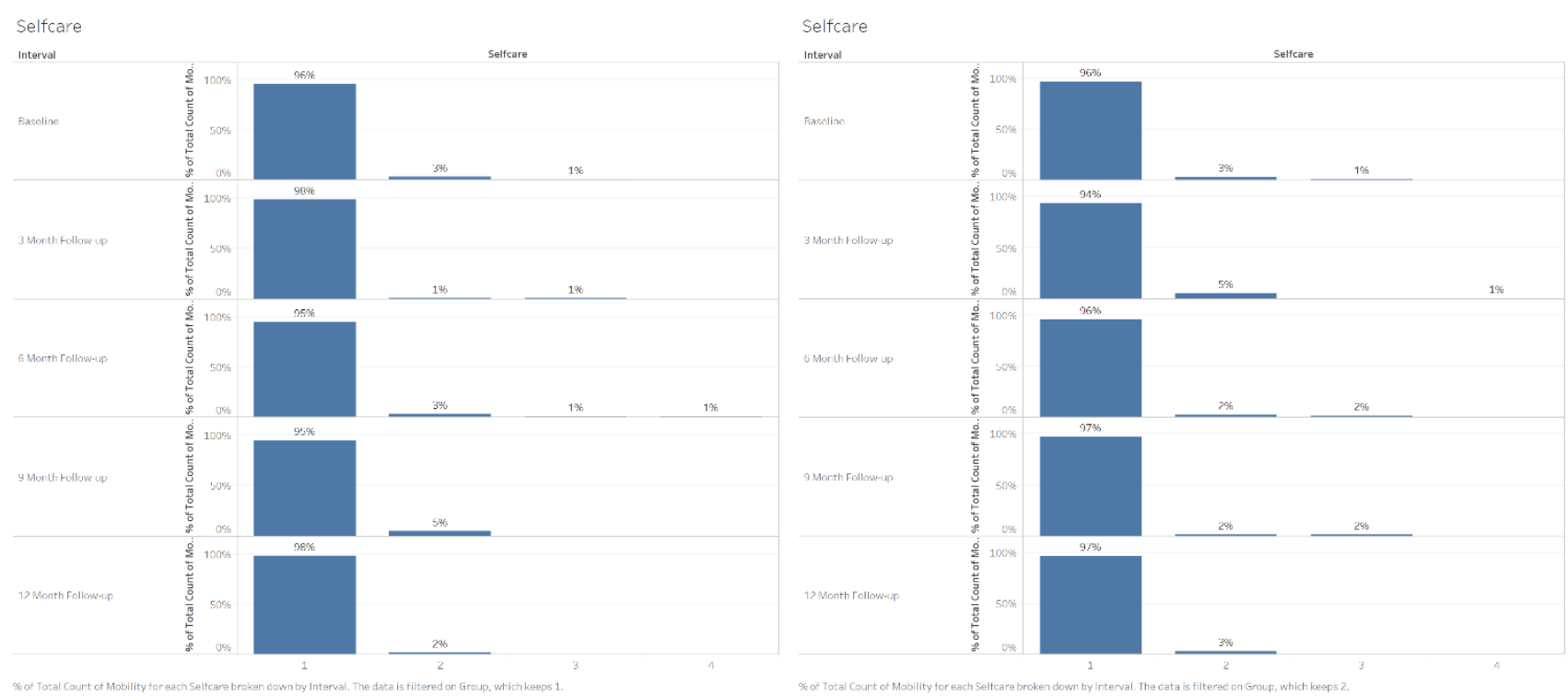

Figure 3. EQ-5D Selfcare dimension by treatment group. Group 1 (BUP/NX) is displayed on the left along with group 2 (MMT) on the right. Very minimal level of improvement is shown in both groups, in the same consistent pattern. 


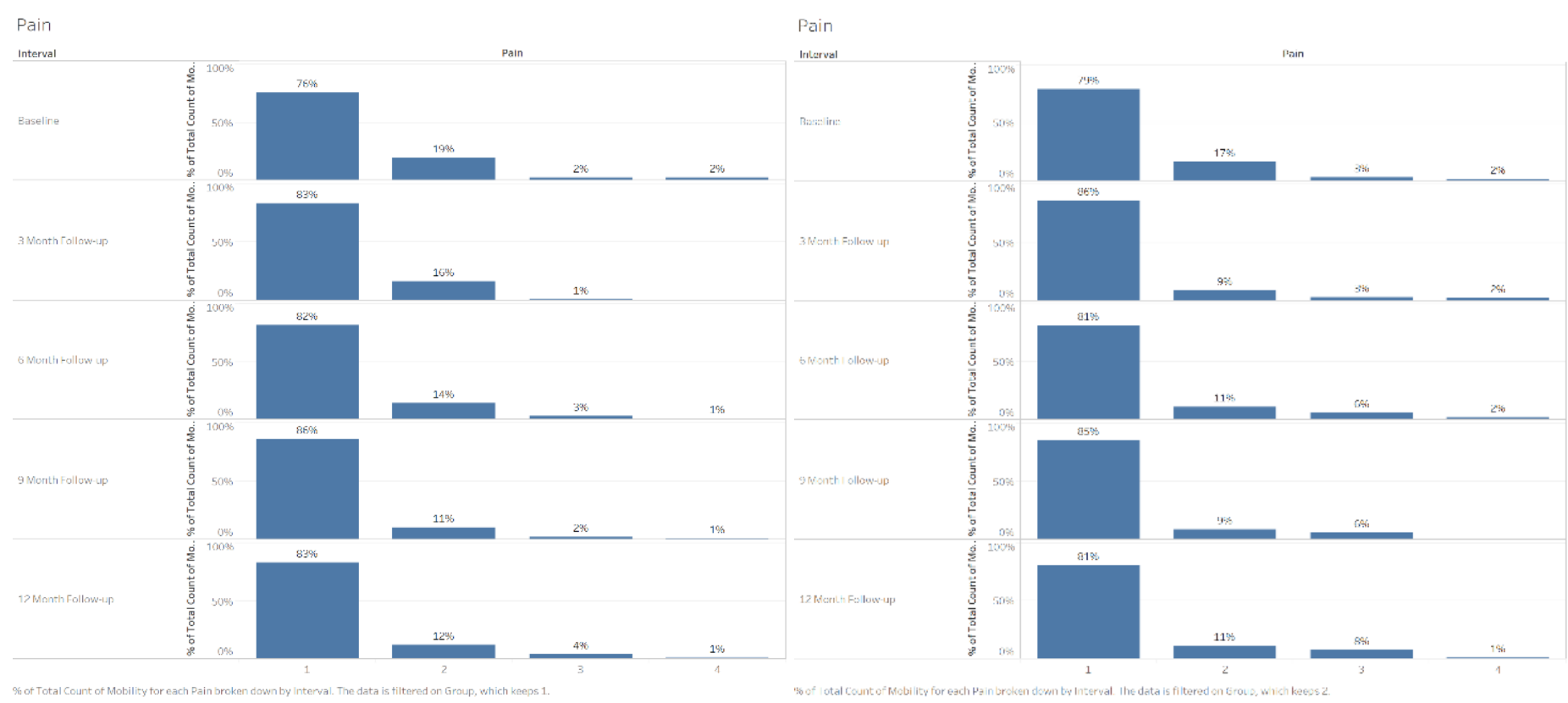

Figure 4. EQ-5D Pain dimension by treatment group. Group 1 (BUP/NX) is displayed on the left along with group 2 (MMT) on the right. Minimal improvement is observed in a quiet consistent manner in the BUP/NX group. The MMT group shows most improvement in the third and ninth months of follow up with a regression to levels close to baseline by the end of the study. The BUP/NX group suggests slightly more favorable results by the end of the study.

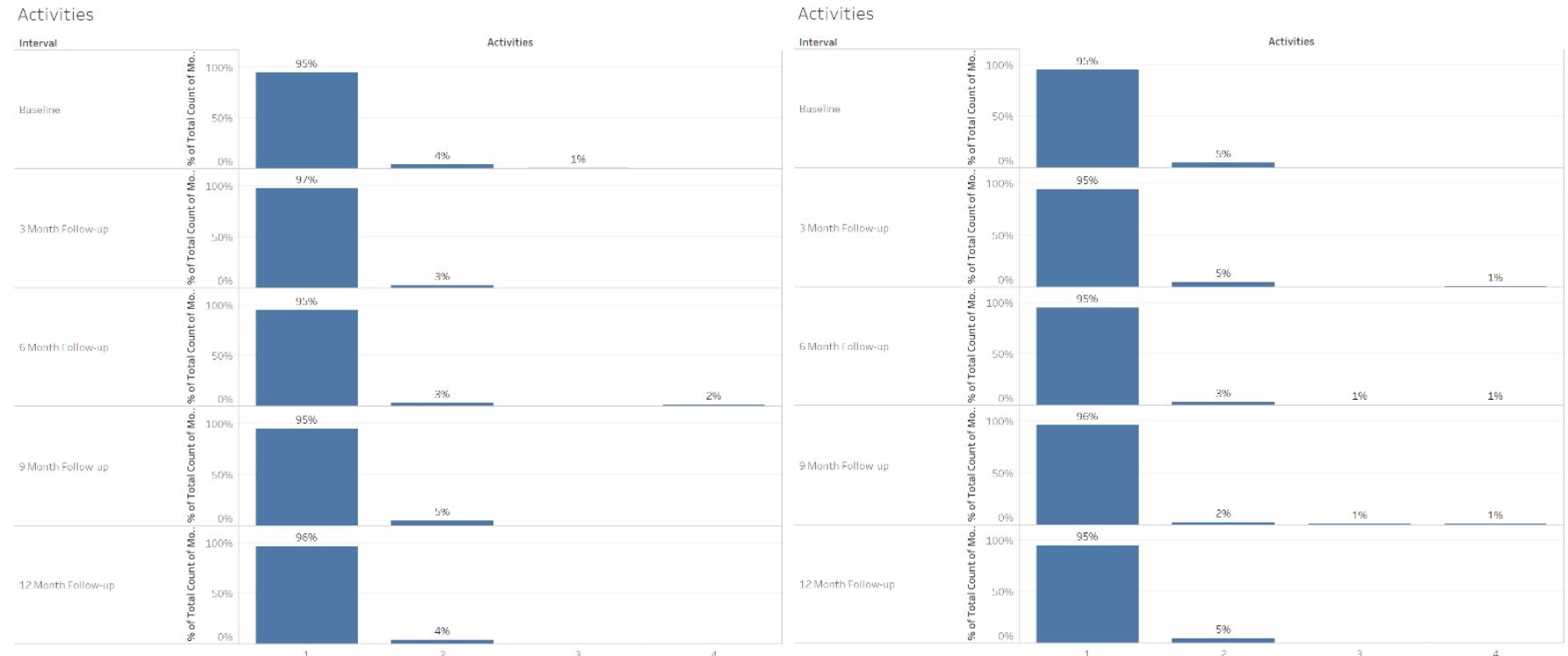

Figure 5. EQ-5D Activities dimension by treatment group. Group 1 (BUP/NX) is displayed on the left along with group 2 (MMT) on the right. There is little change in both group with favorable values from the beginning to the end of the study. 


\section{- Discussion}

QoL for HIV infected individuals with OUD is known to be severely compromised; both BUP/NX and MMT treatment delivery models have been found in improving QoL. ${ }^{12,13,18}$

This study suggests both BUP/NX and MMT treatment delivery models tend to generally improve QoL for the patients of such cohort, but not in all five EQ-5D dimensions. Moreover, not all these improvements seem to be consistent over time. Self-care and activity level were two of the dimensions which did not seem affected by either of the treatment delivery models. A longitudinal study assessing changes in drug use patterns and QoL among a similar population (HIV-infected drug users in Vietnam) partially confirms this outcome as it showed MMT treatment delivery model mostly improved QoL in the psychological dimensions. ${ }^{19}$ Anxiety, mobility, and pain dimensions showed promising results regarding both treatment delivery models.

The MMT group showed steady, yet slight, improvements on anxiety levels. Regarding mobility, this group showed slight improvement towards the middle of the study followed by a regression to levels almost equal to the beginning values. Pain levels in this group were mostly improved in the third and ninth months of follow up with, yet another, regression to levels close to baseline by the end of the study.

The BUP/NX group showed slight improvement in anxiety levels towards the middle of the study followed by a decrease towards the last quarter of the study. Mobility and pain levels in this group improved slightly in a steady manner. Overall, the BUP/NX randomized group demonstrated more favorable improvements in the mobility and pain dimensions while anxiety levels were improved almost equally in both groups. Other studies confirm the effectiveness of BUP/NX in improvement in the pain dimensions by showing "improvements in vitality, social functioning, role physical and emotional, and mental health." 25

The modest improvements in QoL and the diminishing benefits in both treatment models have been shown in another study. ${ }^{14}$ It is recommended that the effectiveness of these treatments has the potential to improve QoL through reduction in drug use and withdrawal symptoms, decreased drug-seeking behavior and increased access to psychosocial and pharmacological treatment for comorbid conditions." ${ }^{15,16}$ As suggested by Volkow and Montaner, there is a possible threshold level to these modes of treatment which is contributed to by "co-occurring mental health conditions, infectious diseases such as HIV and HCV, [and] other chronic medical conditions" in individuals with OUD. ${ }^{17}$

In other studies, although rapid improvement in QoL during the first quarter after applying MMT treatment model was observed; it was decelerated beyond the first three months. ${ }^{18}$ Furthermore, higher dosage of methadone and longer retention were predicted to be associated with higher QoL. ${ }^{18}$ However, another study shows that despite the effectiveness of MMT in "reducing heroin use, injecting practices and crime, and in improving in social functioning and physical symptoms ... the improvement in $[\mathrm{QoL}]$ was not significantly greater as the duration of treatment increased." ${ }^{20}$ For further confirmation, Ponizovsky znc Grinshpoon demonstrated that despite the almost immediate positive results in QoL using an MMT treatment delivery model, the results are short term, yet reach a comparable quality of life quicker than BUP/NX treatment delivery 
model. ${ }^{21}$ Studies seem mixed up on the time span over which the improvement is observed as another study has shown improvement over two to four quarters of MMT. ${ }^{22}$

In comparison with MMT, BUP/NX is thought to have less severe side effects such as less respiratory depression and euphoria while remaining nearly equally effective in decreasing opioid use and is proven to improve QoL in conjunction with psychological counseling. ${ }^{23-26}$

One of the limitations of this study is not being able to control for opioid use after beginning treatment. Opioid use in the long term alters the brain circuitry. ${ }^{27}$ The potential for return to use may explain for multiple cases of loss of initial improvement observed earlier in this study. Furthermore, the study did not consider the role of BUP/NX's "ceiling effect" in the participants' response to the self-reports evaluating the QoL in each treatment model. The decrease in the level of experienced euphoria in the BUP/NX group might have affected their perception of their health related QoL. The data does not generalize to all the patients receiving these forms of treatments. The plateauing trend noted in many of the figures may be due to the QoL of the participants reaching the same level as that of the region's population. Thus, analyses of the EQ-VAS data and normalizing it to the main population's data would provide further insight and help with ruling out some of the limitations. The future studies intend to address these limitations to provide better insight and source for making better judgment regarding the subject matter.

\section{- Conclusion}

The objective of this study was to evaluate the difference in the impact that BUP/NX and MMT treatment delivery models make on the QoL of the HIV-infected participants with OUD in Vietnam. Both treatment delivery models demonstrated the tendency to improve QoL for the HIV-infected individual with OUD living in Vietnam, but not in all dimensions. Self-care and activity level were two of the dimensions which did not seem affected by either of the treatment delivery models. And the BUP/NX randomized group demonstrated more favorable improvements in the mobility and pain dimensions while anxiety levels were improved almost equally in both groups.

\section{- Acknowledgements}

Dr. Todd Korthuis, MD, MPH, Professor of Medicine and Public Health, Addiction Medicine Section Chief, OHSU

Dr. Ryan Cook, PhD, Assistant Professor, Addiction Medicine, OHSU

\section{- Funding}

The BRAVO Trial was supported through grants from the National Institutes of Health National Institute on Drug Abuse (R01DA037441, UG1DA015815). Grant UL1TR002369 provided support of REDCap, the web application this study used for data collection.

NIH Build EXITO: RL5GM118963, UL1GM118963, TL4GM118965

- References 
1."Centers for Disease Control and Prevention." Centers for Disease Control and Prevention, Centers for Disease Control and Prevention.

2. United Nations Office on Drugs and Crime. Vietnam Country Profile 2005. Hanoi: United Nations Office on Drugs and Crime; 2005.

3. Quan VM, Hien NT, Go VF. The HIV epidemic in Vietnam: Past, present, and opportunities. In: Celentano D, Beyrer C, editors. Public Health Aspects of HIV/AIDS in Developing Countries: Epidemiology, Prevention and Care. New York, NY: Springer; 2008. pp. 457-79.

4. Wang H, Wolock TM, Carter A, Nguyen G, Kyu HH, Gakidou E, et al. Estimates of global, regional, and national incidence, prevalence, and mortality of HIV, 1980-2015: the Global Burden of Disease Study 2015. The Lancet HIV 3: e361-e387.

5. Tran, B. X., Nguyen, L. H., Tran, T. T., \& Latkin, C. A. (2018). Social and structural barriers for adherence to methadone maintenance treatment among Vietnamese opioid dependence patients. PloS one, 13(1), e0190941.

6. Nguyen LH, Nguyen HTT, Nguyen HLT, Tran BX, Latkin CA (2017) Adherence to methadone maintenance treatment and associated factors among patients in Vietnamese mountainside areas. Substance Abuse Treatment, Prevention, and Policy.

7. Nosyk B, Anglin MD, Brissette S, Kerr T, Marsh D, Schackman B, Wood E, Montaner JSG. A call for evidence-based medical treatment of opioid dependence in the United States and Canada. Health Aff (Millwood) 2013; 32:1-8.

8. EuroQol Research Foundation. EQ-5D-5L User Guide, 2019.

9. Whelan, Paul J, and Kimberly Remski. "Buprenorphine vs methadone treatment: A review of evidence in both developed and developing worlds." Journal of neurosciences in rural practice vol. 3,1 (2012): 45-50. doi:10.4103/0976-3147.91934

10. Mattick RP, Kimber J, Breen C, Davoli M. Buprenorphine maintenance versus placebo or methadone maintenance for opioid dependence. Cochrane Database Syst Rev. 2003;(2):CD002207. doi :10.1002/14651858.CD002207

11. Millson, P.E., Challacombe, L., Villeneuve, P.J. et al. Self-perceived Health Among Canadian Opiate Users. Can J Public Health 95, 99-103 (2004).

12. Dhawan, A. and A. Chopra (2013). "Does buprenorphine maintenance improve the quality of life of opioid users?" Indian J Med Res 137(1): 130-135.

13. Otiashvili, David et al. "Methadone and buprenorphine-naloxone are effective in reducing illicit buprenorphine and other opioid use and reducing HIV risk behavior--outcomes of a randomized trial." Drug and alcohol dependence vol. 133,2 (2013): 376-82. doi: 10.1016/j.drugalcdep.2013.06.024 
14. Nosyk, B et al. "Short term health-related quality of life improvement during opioid agonist treatment." Drug and alcohol dependence vol. 157 (2015): 121-8. doi:

10.1016/j.drugalcdep.2015.10.009

15. Health Canada. Best Practices: Methadone Maintenance Treatment. 2005 http://www.hcsc.gc.ca/hc-ps/pubs/adp-apd/methadone-bp-mp/index-eng.php.

16. National Consensus Development Panel on Effective Medical Treatment of Opiate Addiction. Effective medical treatment of opiate addiction. JAMA. 1998; 280:1936-1943.

17. Volkow ND, Montaner J. The urgency of providing comprehensive and integrated treatment for substance abusers with HIV. Health Aff (Millwood) 2011; 30:1411-1419.

18. Wang, P. W., et al. (2012). "Change in quality of life and its predictors in heroin users receiving methadone maintenance treatment in Taiwan: an 18-month follow-up study." Am J Drug Alcohol Abuse 38(3): 213-219.

19. Tran, B. X., et al. (2012). "Changes in drug use are associated with health-related quality of life improvements among methadone maintenance patients with HIV/AIDS." Qual Life Res 21(4): 613-623.

20. Teoh Bing Fei, J., et al. (2016). "Effectiveness of Methadone Maintenance Therapy and Improvement in Quality of Life Following a Decade of Implementation." J Subst Abuse Treat 69: 50-56.

21. Alexander M. Ponizovsky \& Alexander Grinshpoon (2007) Quality of Life Among Heroin Users on Buprenorphine versus Methadone Maintenance, The American Journal of Drug and Alcohol Abuse, 33:5, 631-642, DOI: 10.1080/00952990701523698

22. Chou YC, Shih SF, Tsai WD, Li CS, Xu K, Lee TS. Improvement of quality of life in methadone treatment patients in northern Taiwan: a follow-up study. BMC Psychiatry. 2013; 13:190. Published 2013 Jul 16. doi:10.1186/1471-244X-13-190

23. Ling W, Wesson DR. Clinical efficacy of buprenorphine comparisons to methadone and placebo. Drug and Alcohol Dependence. 2003;70(2): S49-S57.

24. Fudala PJ, Bridge TP, Herbert S, et al. Office-based treatment of opiate addiction with a sublingual-tablet formulation of buprenorphine and naloxone. New England Journal of Medicine. 2003;349(10):949-958.

25. Raisch, D. W., et al. (2012). "Health-related quality of life changes associated with buprenorphine treatment for opioid dependence." Qual Life Res 21(7): 1177-1183.

26. Raisch DW, Fye CL, Boardman KD, Sather MR. Opioid dependence treatment, including buprenorphine/naloxone. Annals of Pharmacotherapy. 2002; 36:312-321.

27. Kieffer BL, Evans CJ. Opioid tolerance-in search of the holy grail. Cell. 2002;108(5):587590. doi:10.1016/s0092-8674(02)00666-9 
